\title{
FULLY ATOMISTIC ANALYSIS OF DIFFUSE X-RAY SCATTERING SPECTRA OF SILICON DEFECTS
}

\author{
K. Nordlund, P. Partyka and R. S. Averback
}

Materials Research Laboratory, University of Illinois, Urbana, IL 61801, USA

\begin{abstract}
Diffuse X-ray scattering is a useful method for studying defects in silicon and metals. Although the traditional approaches of analyzing experimental diffuse X-ray scattering data have given much information about the size of defects and defect clusters, they are not very well suited for determining the atomic configuration. We present a fully atomistic computational method to calculate the diffuse X-ray scattering line profile of an arbitrary atomic configuration, and compare line profiles of point defects and Frenkel pair configurations with experiment.
\end{abstract}

\section{INTRODUCTION}

Diffuse X-ray scattering is one of relatively few experimental methods available for studying the atomic structure of defects in crystals. The method is based on measuring the $\mathrm{X}$-ray diffraction lineshape adjacent to a Bragg peak. The interpretation of the measured lineshapes relies on a well-developed continuum theory (see e.g. [1,2]), which has yielded much valuable information on the size and structure of defects in metals [3].

While the continuum theory is able to calculate the dependence of the diffuse scattering on the overall size of a defect cluster, by its very nature as a continuum theory it can not easily be used to predict the diffuse scattering for a specific complex atom configuration. The scattering has been calculated only for the simplest point defects like the dumbbell interstitial in FCC metals. Since defects produced by irradiation in tetrahedral semiconductors are widely believed to be of a complex nature, an atomistic approach to calculating the diffuse scattering could be highly useful in semiconductors.

The X-ray intensity $S$ close to a Bragg peak for a given scattering vector $\mathbf{K}$ for an arbitrary set of atom positions $\mathbf{R}_{i}$ in an infinite monatomic crystal is given by

$$
S(\mathbf{K})=\left|\sum_{i} e^{-\sigma^{2} \mathbf{R}_{i}^{2} / 2 a^{2}} e^{i \mathbf{K} \cdot \mathbf{R}_{i}}\right|^{2},
$$

where $\sigma$ corresponds to the resolution of the experiment $[1,4]$. The diffuse scattering from a defect is determined by the strain field around the defect, which enters the above expression as small displacements in the atom positions $\mathbf{R}_{i}$. In principle, if the atom positions of a defect and all surrounding atoms are known, the scattering can be calculated directly from this expression.

There is of course no way of calculating a sum over an infinite number of arbitrary atom positions. The classical continuum theory overcomes this obstacle by developing approximative expressions for obtaining the strain field and the thus the diffuse scattering around the defect. There is an alternative means, however. While calculating the infinite sum $S(\mathbf{K})$ directly is impossible, doing the sum for merely a very large number of atoms around the defect is usually sufficient to obtain the diffuse scattering with good enough accuracy to allow comparison with experiment [4]. The number of atoms $N$ needed varies 
strongly with the strength of the defect and the experimental resolution $\sigma$. In our experience $N$ usually is of the order of $10^{6}-10^{8}$, which, while large, is not necessarily prohibitive with modern computational capacity and linear algorithms.

In the present Article we present a method by which the diffuse scattering around defects can be calculated directly from expression (1). We limit ourselves to bulk defects with a limited spatial extension. Essentially we calculate the displaced atom positions for a sufficiently large number of atoms around a defect, and use these positions to calculate the sum directly. Our method has the advantage that using the same basic procedure it is possible, at least in principle, to calculate the scattering for an arbitrary atomic configuration. Since the approach is fully atomistic it needs no continuum approximations in calculating the sum 1. It does, however, rely on semi-empirical classical interatomic potentials which are known to be somewhat inaccurate, and therefore can introduce errors in the strain fields and thus the diffuse scattering.

A previous paper by our group has already presented a preliminary version of our method and some experimental results [?]. In the present paper we focus on describing our calculational procedure in detail, then present a few initial results for defect configurations in $\mathrm{Si}$ and compare these with experiment. A more detailed comparison with experimental results will be published later.

\section{CALCULATIONAL PROCEDURE}

To calculate the diffuse scattering we need the displaced positions of a sufficiently large number of atoms surrounding a defect. Computer memory size on typical present-day workstations limits the number of atoms which can be treated efficiently to about 1 million. To treat larger numbers of atoms we have developed an extrapolation process in which the atom coordinates far from the defect center are created using an angular displacement distribution, whereas the (more significant) coordinates close to the defect are taken directly from a relaxed atom cell. The process matches the two kind of defect coordinates smoothly so that no artificial boundary effects occur.

The calculation procedure is fairly complicated and involves several different atom cells. For clarity in the discussion below, these cells are denoted by two uppercase letter codes. The same codes are used in the schematic illustration of the calculation process in Fig. 1.

\section{Atom relaxation}

The initial coordinates for the defect to be studied can be obtained for instance from classical or tight binding molecular dynamics simulations. For simplicity we assume these defects are arranged in a cubic defect (CD) cell and that periodic boundaries were used in the simulation in which they were created.

In the first stage of the calculation process, a spherical defect (SD) cell is created by surrounding the CD cell atoms with atoms at perfect lattice positions out to a radius $R_{S D}$. This spherical shell is relaxed to its energy minimum using a conjugate gradient method [?]. The potential energy between $\mathrm{Si}$ atoms are obtained from the Stillinger-Weber interatomic potential, which is known to reproduce the elastic constants of Si reasonably well $[5,6]$.

Typically we use a sphere with a radius of the order of $100-150 \AA$. For large defects with a strong strain field, a cell with a radius of this order of magnitude may be large enough to obtain the diffuse scattering directly from the SD cell. For small defects, however, this usually is not the case. Therefore, we have developed a method by which the strain field can be extrapolated arbitrarily far out from the defect. 


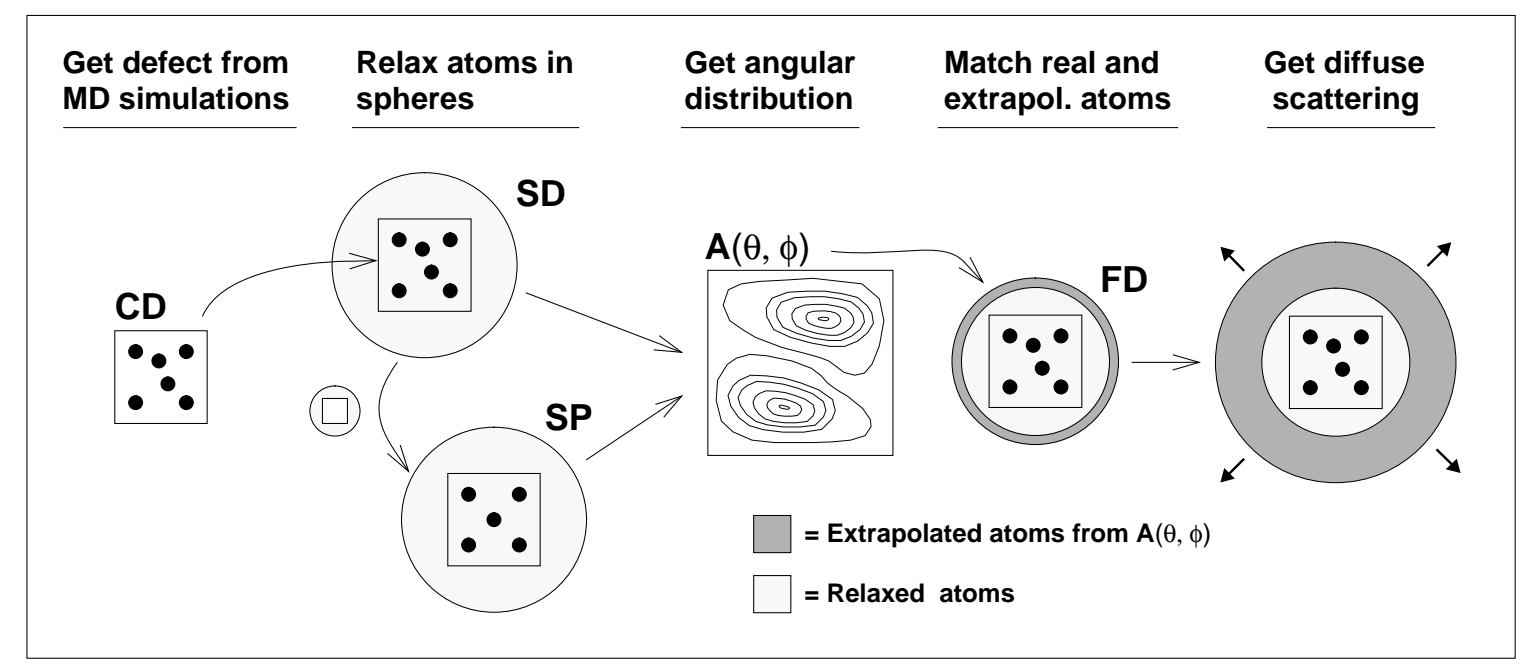

Figure 1: Schematic representation of the calculation procedure used to obtain the diffuse scattering. The leftmost two stages are used to obtain an angular distribution $A(\theta, \phi)$ of the atom displacements (center), which is used to create displaced atom positions outside the sphere with real atom coordinates. These extrapolated atom coordinates are matched with the inner real coordinates before the extrapolation, during which the diffuse scattering is calculated (right). The codes describing the different shells are used in the text.

\section{$\underline{\text { Angular distribution }}$}

To obtain the displacements of the atoms outside the SD cell, we assume that displacements far enough from the defect go down radially as $1 / r^{2}$, a basic tenet of elasticity theory for bulk defects. Then the displacement $d r$ of an atom which is located a distance $r$ from the defect can be written as

$$
r^{2} d r=A(\theta, \phi)
$$

where $\theta$ and $\phi$ define the position of the atom in a spherical coordinate system centered at the defect. The displacement angular distribution $A(\theta, \phi)$ defines the shape of the strain field of any given defect and is independent of $r$ far from the defect center. We note that the assumption that the atom displacements are proportional to $1 / r^{2}$ is not necessary in our method; in principle any functional form $f(r)$ could be used for the $r$ dependence, and even non-radial components could be included in the strain field by making $A$ a vector quantity.

To obtain $A$ we construct a "spherical perfect" (SP) cell by replacing the CD cell defect coordinates in the center of the SD cell with atoms at their ideal Si lattice positions, then relaxing this cell. This way the SP and SD cells will have the same surface relaxation, making surface effects vanish from the displacement field. The angular distribution $A(\theta, \phi)$ is calculated from the displacements of atoms between the SD and SP cells in some $r$ region far from the defect. In practice, $A$ has the form of a discrete matrix with every element giving the average displacements in an angular region $(\Delta \theta, \Delta \phi)$. The displacements for a given $r$ are obtained using two-dimensional interpolation [?]. The validity of the choice of the $r$ region and the defect center position is checked by evaluating $A$ in different $r$ ranges. When the $r$ region is far enough from the center of the defect the angular distribution does not change.

The displacement of an arbitrary atom near the defect can be obtained from the angular distribution. Since we want to use the real atom coordinates close to the defect, we must 
devise a means to ensure that no discrepancy - like a lattice constant or position mismatch occurs between the atom coordinates obtained from $A$ and the real coordinates. Therefore, we create a "fixed defect" (FD) cell by replacing the real atom coordinates in some shell $r_{f} \pm d r_{f}$ in the SD cell with atom coordinates obtained from $A$. By holding the atoms in the $r_{f}$ shell fixed and relaxing the inner real atom coordinates a self-consistent displacement field is created for all $r$. Typically $r_{f} \sim 80 \AA$ and $d r_{f} / 2$ is greater than the potential cutoff. By calculating the scattering for different values of $r_{f}$ it is possible to ensure that the choice of $r_{f}$ does not affect the results.

\section{Calculation of diffuse scattering}

The diffuse scattering is calculated from Eq. 1 using real atom coordinates inside $r_{f}-d r_{f}$ and extrapolated atom coordinates outside this radius. In practice, this is done by creating perfect atom coordinates in shells progressing outwards from the defect center. If the perfect atom position is inside $r_{f}-d r_{f}$ it is replaced by the corresponding real atom in the FD defect cell. Otherwise the atom is displaced with respect to the defect center by a displacement calculated from Eq. 2. By creating the atoms in shells progressing outwards from the center it is easy to determine at what point enough atoms have been created to distinguish the diffuse scattering from the oscillations around the Bragg peak arising from the finite number of atoms. The perfect atom positions are used to obtain the X-ray scattering of a perfect cell.

To obtain the line shape close to a reciprocal lattice vector $\mathbf{G}$, the scattering $S(\mathbf{K})$ is calculated for a set of lattice vectors $\mathbf{K}_{i}$ in the scan direction $\mathbf{K}^{\prime}$

$$
\mathbf{K}_{i}=\mathbf{G}+\mathbf{q}=\mathbf{G}+n \varepsilon \frac{\mathbf{K}^{\prime}}{|\mathbf{G}|}
$$

where $\varepsilon$ is some small number determining the step size around $\mathbf{K}^{\prime}$, and $n$ is an integer. In the present study we treat only radial scans in which $\mathbf{K}^{\prime}=\mathbf{K}$. Since a defect can have a number of orientations with respect to an experimental scan direction, $\mathbf{K}$ and $\mathbf{K}^{\prime}$ have to be obtained for all the equivalent defect orientations and summed with appropriate weighting for comparing a theoretical peak with experiment.

\section{RESULTS AND DISCUSSION}

We present results for six defects in silicon; a monovacancy, a 110 dumbbell interstitial, two Frenkel pairs, and two defect clusters produced by medium-energy cascades. The defects were produced using classical molecular dynamics simulations, the Stillinger-Weber interatomic potential and periodic boundary conditions in all dimensions. One atom in the cell was given a recoil energy to produce the $\mathrm{CD}$ cell used in the diffuse scattering analysis.

The monovacancy had a relaxation volume of $-34 \AA^{3}$ and the dumbbell interstitial $30 \AA^{3}$. Both Frenkel pairs consisted of a vacancy and a dumbbell interstitial. The first was produced by an $18 \mathrm{eV}$ recoil in the open 111 direction, which is the smallest stable Frenkel pair which can be produced with the Stillinger-Weber potential [7]. The interstitial and vacancy are separated by $9.4 \AA$, and the defect has a relaxation volume of $-4 \AA^{3}$. The other Frenkel pair was produced by a $50 \mathrm{eV}$ recoil, has an interstitial-vacancy separation of $11.0 \AA$ and a relaxation volume of $-7 \AA^{3}$.

The first cluster was produced by a $400 \mathrm{eV}$ recoil. It had a relaxation volume of $-19 \AA^{3}$ and a radius of roughly $10 \AA$. The second cluster was produced by a $2 \mathrm{keV}$ recoil. It 


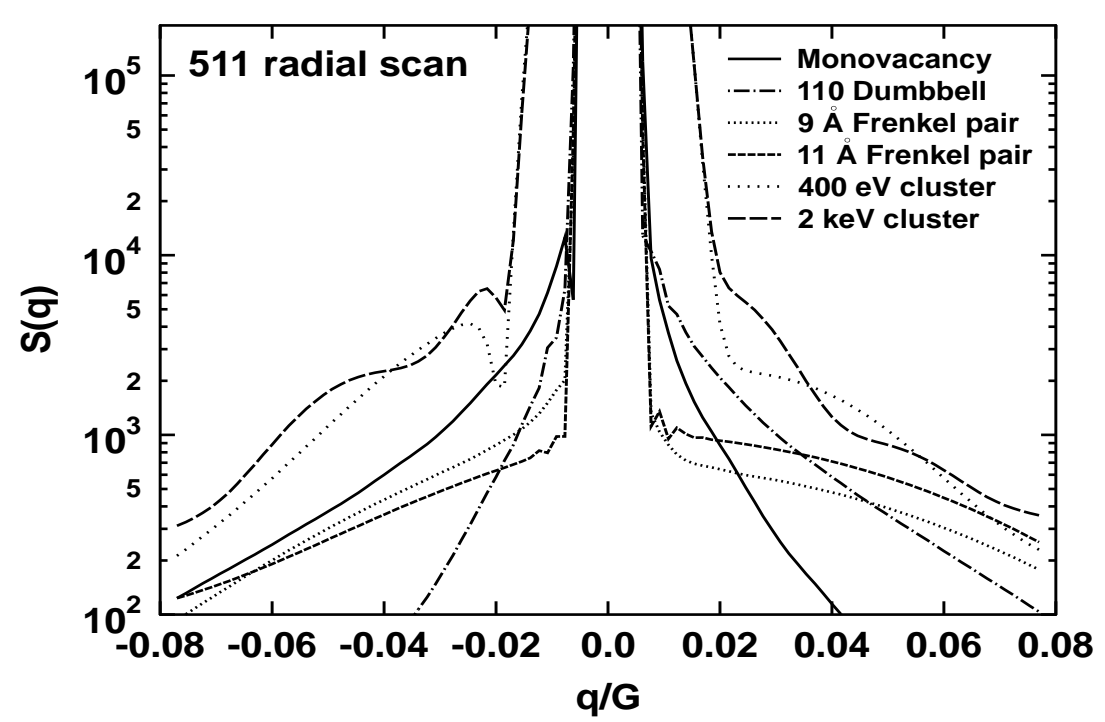

Figure 2: Diffuse scattering from six defects in silicon.

consisted of two defect clusters and a number of isolated defects. The damage radius was about $25 \AA$, and the relaxation volume $-425 \AA^{3}$.

Diffuse scattering results of these defects for the 511 peak with a radial scan are shown in Fig. 2. The scattering from the two clusters is much stronger than that of the Frenkel pairs and the monovacancy, as can be expected from their larger relaxation volumes. It is also interesting to note that the scattering for most of the defects is larger on the side of negative $q$, as can be expected from the negative relaxation volumes. The peculiar shape of the scattering for the two clusters is a consequence of their complex shape.

It is interesting to note that the scattering from the interstitial and vacancy are almost perfect mirror images of each other, reflecting the opposite sign but almost equal magnitude of their relaxation volumes. The symmetric part of the monovacancy scattering deviates somewhat from ideal $1 / q^{2}$ depedence for large $q$ (Fig. 3). We do not yet know the reason of this deviation, but suspect it may be due to the approximations involved in the interpolation of the angular distribution.

In Fig. 3 we show experimental data of symmetric (Huang) diffuse scattering from defects produced by $2.5 \mathrm{MeV}$ electron irradiation [8], along with the symmetrisized scattering from the monovacancy, interstitial, and two Frenkel pairs. Since $2.5 \mathrm{MeV}$ electrons are not expected to produce complex damage at low doses, the cluster data is not included in the figure. The scattering shown is in units of $S q^{2}$ to remove the strong $1 / q^{2}$ decrease of the actual scattering. The (very small) scattering from the perfect cell has been subtracted from the results. The intensity ratio between the experimental and theoretical data is a free parameter which has been adjusted to provide a reasonable fit to the data. From the figure it would appear that close Frenkel pairs agree fairly well with the rise in the scattering. The constant part at small $q$ could arise from a background of point defects or other damage.

\section{CONCLUSIONS}

A method to calculate the diffuse scattering from an arbitrary atomic defect configuration has been presented. It was used to calculate the diffuse scattering from different defect configurations in silicon, and the results were compared with experimental data. 


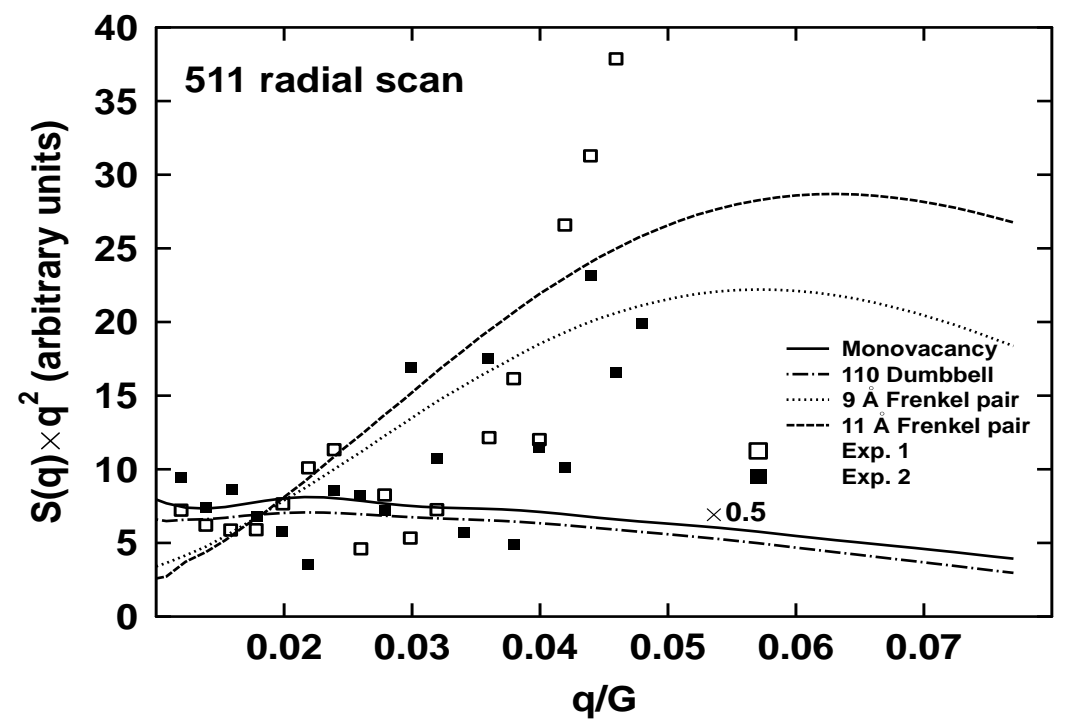

Figure 3: Symmetric part of the diffuse scattering multiplied by $q^{2}$ for a monovacancy, an interstitial and two Frenkel pairs, and experimental data of defects produced by 2.5 $\mathrm{MeV}$ electron irradiation from [8]. The monovacancy and interstitial scattering has been multiplied by 0.5 .

\section{ACKNOWLEDGEMENTS}

We thank Dr. P. Ehrhart for valuable comments during the course of this work. The research was supported by the US Department of Energy, Basic Energy Sciences under grant DEFG02-91ER45439. Grants of computer time from the National Energy Research Computer Center at Livermore, California, and the National Center for Supercomputing Applications at Urbana-Champaign are gratefully acknowledged.

\section{REFERENCES}

1. P. H. Dederichs, J. Phys. F: Metal Phys. 3, 471 (1973).

2. H. R. Schober and K. W. Ingle, J. Phys. F: Metal Phys. 10, 575 (1980).

3. P. Ehrhart, K. H. Robrock, and H. R. Shober, in Physics of Radiation Effects in Crystals, edited by R. A. Johnson and A. N. Orlov (Elsevier, Amsterdam, 1986), p. 3.

4. D. T. Keating and A. N. Goland, Acta Cryst. A 27, 134 (1971).

5. F. H. Stillinger and T. A. Weber, Phys. Rev. B 31, 5262 (1985).

6. H. Balamane, T. Halicioglu, and W. A. Tiller, Phys. Rev. B 46, 2250 (1992).

7. M.-J. Caturla, T. Diaz de la Rubia, and G. H. Gilmer, Mat. Res. Soc. Symp. Proc. 316, 141 (1994).

8. H. Zillgen and P. Ehrhart, Nucl. Instr. Meth. Phys. Res. B 127-128, 27 (1996). 